\title{
ACTIVIDAD DE COLINESTERASA PLASMÁTICA Y SINTOMA- TOLOGÍA PRESENTE EN FUMIGADORES DEL VALLE DE MALA, EXPUESTOS A PLAGUICIDAS ANTICOLINESTERÁSICOS
}

\author{
C. Huamaní-Pacsi, R.P. Sánchez-Ramírez, H.C. Cataño , R. Huguet-Tapia, E. Carranza
}

Laboratorio de Biología Molecular. Instituto de Química Biológica, Microbiología y Tecnología. Facultad de Farmacia y Bioquímica

\begin{abstract}
RESUMEN
Debido a que investigaciones previas han reportado que la exposición a plaguicidas anticolinesterásicos (órganofosforados y carbamatos) a dosis repetidas por largos periodos de tiempo producen neurotoxicidad, se determinó la actividad de Colinesterasa plasmática y la sintomatología presente en 83 fumigadores del valle de Mala-Perú, con la finalidad de conocer los niveles de exposición y tener un indicio del efecto en la salud de los fumigadores. Se utilizó comø grupo control a 28 sujetos emparejados con las características sociodemográficas de los fumigadores, pero cuya activiđad fue distinta a la agricultura. Se encontró que los fumigadores estudiados no cumplieron con las medidas de protección durante la manipulación de plaguicidas. La media de la actividad de colinesterasa plasmática en 4 de 5 grupos formados según el lugar de procedencia de les fumigadores fue significativamente menor que la media del grupo control. Los 5 síntomas que más se presentaron en los fumigadores fueron cefalea $(62 \%)$, visión borrosa $(52 \%)$, vértigo (37\%), salivación (32\%) y sudoración $(31 \%)$. Con los resultados encontrados en los fumigadores, se concluye que sus niveles de exposición a plaguicidas anticolinesterásicos varían ampliamente y que sus síntomas estuvieron relacionados con su ocupación.
\end{abstract}

Palabras clave: Organofosforados, carbamatos, fumigadores, colinesterasa plasmática, toxicología.

\begin{abstract}
SUMMARY
As previous studics have reported that long-term exposure to pesticides antichtlinesterases (organophosphates and carbamates) at repeated dose produces neurotoxicity, it has determinated the plasma cholinesterase activity and the symptomatology in $\mathbf{8 3}$ pesticides applicators from vallcy of Mala-Perú with the purpose to knowing the exposure levels and to have an indication of the health effects in the applicators. The control group consisted of 28 subjects who were matched with the sociodemographics characteristics of the applicators but whose economical activity was different to agriculture. It was found that the studied applicators did not fulfill the protection rules during the manage of pesticides. The mean of plasma cholinesterase activity in 4 from 5 formed groups by the proccedence place of applicators were significatively lower than the mean of the control group. The 5 symptoms more found were headache (62\%), blurred vision (52\%), dizziness $(37 \%)$, salivation $(32 \%)$ y sweating $(31 \%)$. With the found results in the studied applicators, we conclude that their exposure levels to pesticides anticholinesterases vary widely and their symptoms were related to their occupation.
\end{abstract}

Key words: OTganophosphorus, carbamates, applicators, plasma cholinesterase, toxicology.

\section{INTRODUCCIÓN}

Los plaguicidas son utilizados en actividades de salud pública, comercio, uso doméstico y principalmente en la agricultura, con el fin de prevenir, destruir, repeler o atenuar alguna plaga, que puede ir desde insectos, animales, hierba mala hasta microorganismos (1). Debido a su amplio uso, es posible que casi todos tengamos un antecedente de exposición, pero, probablemente, los trabajadores agrícolas son los que más expuestos están a los plaguicidas. En el mercado mundial, existen un gran número de plaguicidas que pueden ser agrupados según su origen químico como: plaguicidas organofosforados, carbamatos, organoclorados, piretroi- des, derivados del bipiridilo, triazinas, tiocarbamatos, derivados del ácido fenoxiacético, derivados de la cumarina, derivados del cloronitrofenol, compuestos organomercuriales, entre otros. De éstos, los plaguicidas organofosforados y carbamatos son los más utilizados a nivel mundial y a su vez, los más tóxicos $(2,3)$. Su toxicidad recae principalmente sobre el sistema nervioso, inhibiendo la acetilcolinesterasa neuronal, produciendo un incremento de la acetilcolina en las sinapsis y desencadenando un síndrome colinérgico agudo vía una neurotransmisión continua, que puede llevar a la muerte si no existe una apropiada y rápida intervención (4). Aunque ambos inhiben la acetilcolinesterasa, los plaguicidas organofosforados son considerados 
más tóxicos que los carbamatos. Esto se debe a que los organofosforados inhiben la acetilcolinesterasa de manera irreversible mientras que los carbamatos lo hacen de manera reversible (2). Por otro lado, numerosos estudios otorgan mayores evidencias de la alta toxicidad de los plaguicidas organofosforados. Se ha reportado que luego de $24-96$ horas de una crisis colinérgica aguda, se puede experimentar un síndrome intermedio, caracterizado por paresia aguda del músculo respiratorio, paresia de los miembros proximales y músculos faciales, produciéndose además parálisis de los nervios craneales $(5,6)$. También se ha reportado que después de dos o tres semanas del episodio agudo producido, puede ocurrir una neuropatía retardada (OPIDN, organophosphorus induced delayed neurotoxicity). Los rasgos más comunes de este síndrome recaen sobre el sistema nervioso central y periférico produciéndose una neuropatía mixta sensorio-motriz $(7,8)$. El mecanismo del síndrome intermedio es incierto (4), mientras que el mecanismo de la neuropatía retardada sería por la inhibición de una enzima llamada esterasa neurotóxica (NTE, neuropahły target esterase) (9). Además, recientemente, se ha reportado que la exposición crónica a organofosforados produce neurotoxicidad $(9,10,11)$, caracterizada con el descenso persistente de la funcionalidad neuropsicológica (incluyendo la atención acústica, atención verbal, memoria visual, velocidad visomotora, secuenciación y solución de problemas, estabilidad motora, reacción y destreza). Por lo mencionado anteriormente, se puede deducir que entre los trabajadores agrícolas, aquellos que continuamente estấn aplicando (fumigando) plaguicidas organofosforados y carbamatos representan el grupo de mayor riesgo. Este riesgo aumenta cuando no se tiene el entrenamiento adecuado para la aplicación de plaguicidas, ni se cumple con las medidas de protección necesarias durante su manipulación.

La evaluación del riesgo de toxicidad hacia plaguicidas organofosforados y carbamatos involucra la determinación de indicadores biológicos de exposición, los cuales pueden servir como una alarma previa a la aparición de las manifestaciones clínicas (12). La Organización Mundial de la Salud (OMS) sugiere la medición de la actividad de colinesterasa plasmática como indicador biológico de exposición, así, una disminución de la actividad de la colinesterasa plasmática de un $30 \%$, indicaría una exposición baja y disminuciones mayores al $50 \%$ indicarían una alta exposición relacionada a una intoxicación. aguda (13).
El presente trabajo tiene como objetivo conocer las medidas de protección utilizadas en la manipulación de plaguicidas anticolinesterásicos, asî como la actividad de la colinesterasa plasmática y la sintomatología presente de un grupo de fumigadores del valle de Mala, Lima-Perú.

\section{MATERIALES Y MÉTODOS}

\section{SELECCIÓN DE LA POBLACIÓN Y RECOLEC- CIÓN DE MUESTRAS}

El valle de Mala, situado en la provincia de Cañete (Departarnento de Lima), es la principal productora de manzanas del Perú. El clima, la variedad y la posibilidad de producir una floración pareja, permite a los agricultores de este valle obtener 3 cosechas en dos años, sobrepasando el ritmo habitual de una cosecha anual. Esta intensificación del cultivo también ha incrementado la utilización de plaguicidas en esta zona.

El grupo utilizado como muestra estuvo compuesto por fumigadores de 2 distritos de la provincia de cañete: Mala (Santa Enriqueta) y Calango con sus poblados: Calango, La Capilla, La Vuelta y Correviento, localizados en la parte media y baja del valle de Mala. 83 fumigadores de ambos sexos (70 hombres y 13 mujeres), cuyas edades estuvieron comprendidas entre 17 a 87 años, fueron contactados para participar en el estudio. Entre los 5 plaguicidas más usados por los fumigadores estudiados fueron Dimetoato (organofosforado) en 79\%, Dinitro-orto-cresol en $77 \%$, aceite mineral en $56 \%$, Metomilo (carbamato) en $46 \%$ y Metamidofos (organofosforado) en $40 \%$.

El grupo control estuvo conformado por $28(22$ hombres y 6 mujeres) pobladores de la misma zona geografica que los fumigadores estudiados, pero con la característica de que su actividad económica no estuvo relacionada con la agricultura $y$, por lo tanto, sólo tuvieron el potencial de exposición a plaguicidas propios de la comunidad.

A ambos grupos se les aplicó una encuesta que constaba de dos partes. En la primera parte se recogieron datos de filiación, grado de instrucción, hábitos alimenticios y de higiene personal, además de un interrogatorio sobre las medidas de protección empleadas en la manipulación de los plaguicidas. La segunda parte de la encuesta estuvo dirigida a la búsqueda de síntomas que nos dieran un indicio de 
una leve intoxicación aguda o sospecha de intoxicación crónica.

De cada participante se extrajo $5 \mathrm{~mL}$ de sangre en tubos vacutainer con anticoagulante EDTA. Las muestras se centrifugaron a $2500 \mathrm{RPM}$ durante 5 minutos, conservándose solamente el plasma para el análisis. Las muestras fueron transportadas a $4^{\circ} \mathrm{C}$ hasta el laboratorio donde se congelaron a $-20^{\circ} \mathrm{C}$ hasta su posterior análisis.

\section{DETERMINACIÓN DE LA COLINESTERASA PLASMÁTICA}

La actividad de la colinesterasa plasmática se determinó espectrofotométricamente según el método de Ellman y col. (14), utilizando yoduro de acetiltiocolina (Sigma), como sustrato. Se adicionó 50uL de plasma a $3 \mathrm{~mL}$ de una solución de ácido ditiobisnitrobenzoico (Sigma) que estuvo en buffer fosfato $0.1 \mathrm{M} \mathrm{pH}=7.7$. A ésta última mezcla se adicionó $20 \mathrm{uL}$ del reactivo yoduro de acetiltiocolina $0.075 \mathrm{M}$. Se mezcló bien y se realizó la lectura a $412 \mathrm{~nm}$ en un espectrofotómetro Spectronic 20 . Sc registró la lectura cada minuto durante un periodo de tres minutos a $25^{\circ} \mathrm{C}$. Para el blanco se realizó el mismo procedimiento anterior, pero sin la adición del plasma. Se calculó el promedio de las tres lecturas (absorbancia promedio por minuto) para determinar la actividad enzimática en $\mathrm{mU} / \mathrm{mL}$.

\section{ANÁLISIS ESTADÍSTICO}

Para el tratamiento de los datos se utilizó el paquete estadístico SPSS versión 11.0. Se elaboraron medidas de tendencia central y dispersión para la descripción de la información. Para evaluar la diferencia entre los grupos se utilizó el ensayo $t$-student y el análisis de variancia (one-way ANOVA).

\section{RESULTADOS}

Características antropométricas de los participantes. La edad promedio de los fumigadores fue de $\mathbf{4 2 . 3 8}$ años con una desviación estándar (D.E.) de 18.76 y una mediana de 40.00 . El pesa y la talla promedio fueron de $67.46 \mathrm{Kg}$ (D.E.=12.37) y $1.62 \mathrm{~cm}$ (D.E = $0.08)$, respectivamente.

El grupo control estuvo emparejado con las características de los fumigadores. Así, su edad promedio fue de 42.00 (D.E $=11.73$ ) y su mediana de 43.00 . El peso y la talla promedio de $65.60 \mathrm{~kg}$ (D.E. $=9.51)$ y $1.63 \mathrm{~cm}$ (D.E $=0.09)$, respectivamente.

Condiciones de trabajo y hábitos de higiene. La antigüedad promedio, fumigando, fue de 15 años (D.E.=14). El $74.1 \%$ de los fumigadores tuvo entre 0 a 20 años de antigüedad, el $18.8 \%$ entre 21 a 41 años $y$ el $7.1 \%$ entre 42 a 63 años. Los fumigadores refirieron realizar al menos 3 campañas de fumigación al año. El número de aplicaciones promedio en la última campaña según la antigüedad fue: 4 aplicaciones en el grupo de 0 a 20 años, 6 en el de 21 a 41 años y 5 en el de 42 a 63 años.

El $60 \%$ de los fumigadores no tuvo ninguna clase de asesoria en el manejo y aplicación de plaguicidas. La mayoría de fumigadores $(80 \%)$ refirió preparar la mezcla antes de la aplicación y el equipo más utilizado para la aplicación fue la mochila $(54 \%$ de los fumigadores).

El $80 \%$ de los fumigadores dijo haber tenido contacto dérmico (se moja) con los plaguicidas cuando realizan la aplicación. Como se puede observar en la Tabla 1, las indumentarias más usadas por los fumigadores para su protección durante la mezcla y/ o aplicación fueron: pantalón $(92 \%)$, camisa $(90 \%)$, zapatos $(62 \%)$ y gorra o sombrero (55\%). En cuanto a las medidas higiénicas de los fumigadores, el $77 \%$ refirieron bañarse después de fumigar, el $39 \%$ refirió cambiarse la ropa empleada en la fumigación o el uniforme diariamente y el $27 \%$ refirió consumir alimentos en el lugar de mezcla y/o aplicación.

Tabla 1. Protección usada por el fumigador cuando realiza la aplicación de plaguicidas.

\begin{tabular}{lcc}
\hline \multicolumn{1}{c}{ Protección } & $\begin{array}{c}\text { N. }^{\circ} \text { de } \\
\text { Trabajadores }\end{array}$ & Porcentaje \\
\hline Camisa & 80 & $92 \%$ \\
Pantalón & 78 & $90 \%$ \\
Zapatos & 62 & $71 \%$ \\
Sombrero/gorra & 48 & $55 \%$ \\
Máscara & 25 & $29 \%$ \\
Pañuelo & 21 & $24 \%$ \\
Guantes & 17 & $20 \%$ \\
Espaldar & 17 & $20 \%$ \\
Delantal & 13 & $15 \%$ \\
Botas & 12 & $14 \%$ \\
Overol & 8 & $9 \%$ \\
Protectores de ojos & 1 & $1 \%$ \\
\hline
\end{tabular}


Tabla 2. Medias de la actividad de colinesterasa plasmática según procedencia.

\begin{tabular}{lcccccc}
\hline \multicolumn{1}{c}{ Enzima } & $\begin{array}{c}\text { Santa Enri- } \\
\text { queta } \\
(\mathbf{n = 1 6 )}\end{array}$ & $\begin{array}{c}\text { La Capilla } \\
(\mathbf{n}=\mathbf{1 7})\end{array}$ & $\begin{array}{c}\text { La Vuelta } \\
(\mathbf{n}=\mathbf{1 6})\end{array}$ & $\begin{array}{c}\text { Calango } \\
(\mathbf{n}=\mathbf{1 7})\end{array}$ & $\begin{array}{c}\text { Correviento } \\
(\mathbf{n = 1 7 )}\end{array}$ & $\begin{array}{c}\text { Control } \\
\text { V(n= 28) }\end{array}$ \\
\hline ChE & $1124.49 \pm$ & $1137.29 \pm$ & $1119.36 \pm$ & $1106.41 \pm$ & $1117.71 \pm$ & $1221.68 \pm$ \\
(Media \pm D.E.) & 132.18 & 188.53 & 154.19 & 153.05 & 168.20 & 44.80 \\
*Probabilidad & $<0.02$ & n.s. & $<0.02$ & $<0.01$ & $<0.05$ & - \\
(P) & $<$ & & &
\end{tabular}

n.s.: no significativo. ${ }^{*}$ En relación al grupo control.

Indicador de exposición. Se utilizó como indicador de exposición la actividad de la colinesterasa plasmática. Los valores medios de actividad de colinestersa plasmática de los fumigadores y controles fueron: 1121,03 U/L (Rango: 654.6-1426.6 y D.E.= 151.80) y 1221.68 U/L (Rango: 1145.43-1277.43 y D.E. $=44.80)$, respectivamente. Al comparar estas medias por el ensayo de $t$-student, no se encontró diferencia significativa, pero al agrupar a los fumigadores según su lugar de procedencia y al comparar las medias de la actividad de colinesterasa plasmática de cada grupo con la media del grupo control, se encontró diferencia significativa en 4 de los 5 grupos formados (Tabla 2).

\section{Sintomatología}

Los 5 síntomas que se presentaron con mayor frecucncia fueron: cefalea en 54 fumigadores; visión borrosa en 45; vértigo en 32; salivación en 28 y sudoración en 27 (Tabla 3).

Tabla 3. Síntomas presentes en fumigadores.

\begin{tabular}{|l|c|c|}
\hline \multicolumn{1}{|c|}{ Síntomas } & $\begin{array}{c}\mathbf{N}^{\circ} \text { de } \\
\text { Trabajadores }\end{array}$ & Porcentaje \\
\hline Cefalea & 54 & $62 \%$ \\
\hline Visión borrosa & 45 & $52 \%$ \\
\hline Vértigo & 32 & $37 \%$ \\
\hline Salivación & 28 & $32 \%$ \\
\hline Sudoración & 27 & $31 \%$ \\
\hline Debilidad & 25 & $29 \%$ \\
\hline Náuseas & 18 & $21 \%$ \\
\hline Intranquilidad & 18 & $21 \%$ \\
\hline Pérdida del apetito & 17 & $20 \%$ \\
\hline Dolor estomacal & 17 & $20 \%$ \\
\hline Fasciculaciones & 17 & $20 \%$ \\
\hline Inestabilidad al & 16 & $18 \%$ \\
\hline caminar & 10 & $11 \%$ \\
\hline Sensación de miedo & 9 & $10 \%$ \\
\hline Vómito & 6 & $7 \%$ \\
\hline Diarrea & 6 & $7 \%$ \\
\hline Cianosis & & \\
\hline
\end{tabular}

Para observar si la aparición de la sintomatología estuvo relacionada con la actividad de colinesterasa plasmática, se agrupó a los fumigadores según el número de síntomas que presentaban, así se obtuvo 3 grupos: de 1 a 3 síntomas, de 4 a 7 y de 8 a más, luego se comparó las medias de la actividad de colinesterasa de los fumigadores de cada grupo. $\mathrm{El}$ análisis estadístico reveló que no hubo diferencia significativa entre los tres grupos (tabla 4).

Tabla 4. Media de la actividad de la colinesterasa estratificada según el número de síntomas.

\begin{tabular}{|l|c|c|c|}
\hline $\begin{array}{c}\text { Número de } \\
\text { síntomas }\end{array}$ & $\mathbf{n}$ & $\begin{array}{c}\text { ChE } \\
\text { (Media } \pm \text { D.E.) }\end{array}$ & $p^{\mathbf{a}}$ \\
\hline 1 a 3 & 47 & $1139,82 \pm 115,13$ & \\
\cline { 1 - 2 } 4 a 7 & 31 & $1108,72 \pm 184,65$ & \multirow{2}{*}{0.233} \\
\hline 8 a más & 5 & $1024,47 \pm 211,89$ & \\
\hline
\end{tabular}

a. One-way ANOVA. p. Significancia.

\section{DISCUSIÓN}

En el caso de los fumigadores, la exposición a plaguicidas organofosforados y carbamatos ocurre principalmente durante su mezcla y aplicación. Además, se conoce que estas sustancias cuando se ponen en contaclo con el cuerpo pueden ingresar por diversas rutas como: vía inhalatoria, absorción por la piel, absorción por la conjuntiva ocular o por ingestión (3). Por ello, resulta difícil evitar del todo su ingreso y más bien lo que se busca es disminuir el ingreso de estas sustancias a niveles que ro representen riesgo para la salud, reduciendo la exposición. Existen varias medidas que contribuyen a reducir la exposición como tener el entrenamiento adecuado para la mezcla y aplicación de plaguicidas, usar la indumentaria apropiada mientras se manipulan los plaguicidas, así como tener el descanso apropiado luego de una campaña de aplica- 
ción, que puede consistir simplemente en rotar al fumigador a otras labores, manteniéndolo alejado de los plaguicidas ${ }^{(15)}$.

Los resultados de nuestro estudio indican que los fumigadores estudiados del Valle de Mala no cumplieron con las medidas adecuadas para reducir la exposición. Así, el $60 \%$ de ellos refirió no tener ninguna clase de asesoría en el manejo y aplicación de plaguicidas. Por ello, es probable que los fumigadores con menos años de antigüedad tengan más riesgo de sufrir intoxicaciones agudas que los de mayor antigüedad debido a su inexperiencia en la manipulación de plaguicidas. En cuanto a la protección durante la mezcla y aplicación de plaguicidas, los fumigadores refirieron usar pantalón $(92 \%)$, camisa $(90 \%)$, zapatos $(62 \%)$ y gorra o sombrero $(55 \%)$. Sin embargo, estas prendas no aportan la suficiente protección como lo hacen los uniformes, que están diseñados para estas actividades. No obstante, se conoce que, a pesar de utilizar la indumentaria adecuada, una pequeña proporción de plaguicidas pueden atravesarlos y ponerse en contacto con el cuerpo (16); por ello, una medida higiénica destinada a reducir este contacto es bañarse luego de la fumigación. Sin embargo, en los fumigadores estudiados un porcentaje bajo pero considerable $(23 \%)$ refirió no bañarse después de la fumigación. Otra medida higiénica consiste en cambiarse el uniforme diariamente. Sin embargo, de los fumigadores estudiados, sólo el $39 \%$ refirió cambiarse la ropa empleada en la fumigación o el uniforme diariamente. Algo más que puede disminuir el contacto con los plaguicidas es no consumir alimentos en los sitios de mezcla y/o aplicación. Aunque, sólo el $27 \%$ de los fumigadores estudiados refirieron consumir alimentos en los sitios de mezcla y/o aplicación, estas prácticas aún continúan siendo comunes en un sector de fumigadores del país.

De los resultados encontrados en la encuesta laboral, se puede deducir que existe mucha variación en los niveles de exposición a plaguicidas organofosforados y carbamatos entre los fumigadores estudiados, lo cual se pude confirmar al observar los valores máximo $(1426,6 \mathrm{U} / \mathrm{L})$ y mínimo $(654,6$ $\mathrm{U} / \mathrm{L}$ ) del indicador de exposición. Por otro lado, esta variación en los niveles de exposición, puede explicar en parte porque no se encontró diferencia significativa entre la actividad de colinesterasa del grupo control y los fumigadores cuando se les consideró como un solo grupo. Algo que apoya esto es que cuando se agrupó a los fumigadores según su lugar de procedencia y se les comparó con el grupo control, se encontró diferencia significativa en la actividad de colinesterasa en 4 de los 5 grupos formados. Al parecer, al agrupar a los fumigadores según su lugar de procedencia, se redujo la variación en la exposición debida a conductas de protección propias de los fumigadores de cada poblado. Esto puede sugerir que en estudios posteriores para efectos comparativos podría agruparse a los fumigadores según niveles o gradientes de exposición. Así, se podría agrupar a los fumigadores en grupos de alta, intermedia y baja exposición basados en sus conductas de protección y tener resultados más representativos de cada grupo.

Aunque la encuesta clínica no fue validada previamente, la sintomatología presente en los fumigadores (tabla 3) da un indicio que la exposición a plaguicidas anticolinesterásicos estuvo afectando a la salud de los fumigadores. Como se sabe, es de esperar que exista una correspondencia entre la actividad de colinesterasa y la severidad de la sintomatología. Sin embargo, algunos estudios han reportado que trabajadores ocupacionalmente expuestos a plaguicidas con niveles de colinesterasa normales o deprimidos dentro de los límites normales, presentaron sintomatología general e inespecífica relacionada con su exposición a plaguicidas organofosfo$\operatorname{rados}^{(17,18,19,20,21)}$ así como también se ha reportado lo contrario, trabajadores con niveles de colinesterasa deprimidos fuera de los límites normales no presentaron ninguna sintomatologia ${ }^{(22,23)}$. Esto puede deberse a la falta de una relación significativa entre la dosis y respuesta como lo han señalado investigaciones previas ${ }^{(24,25)}$. En el presente estudio, si bien no se encontró diferencia significativa al agrupar a la actividad de colinesterasa de los fumigadores según el número de síntomas que presentaron, se puede notar que el grupo de fumigadores con 8 a más síntomas tienen el valor de colinesterasa en promedio menor que el grupo con 4 a 7 síntomas, y a su vez, éste tiene el valor de colinesterasa menor que el grupo con 1 a 3 síntomas. Esto sugiere que debe estudiarse aun más la relación entre la sintomatología de los plaguicidas anticolinesterásicos y los niveles de colinesterasa.

\section{CONCLUSIONES}

1. Las medidas de protección de los fumigadores estudiados frente a la exposición de plaguicidas anticolinesterásicos son escasas, así como también algunas medidas higiénicas. 
2. Debido a que cada fumigador asume una conducta propia de protección, existe mucha variación en los niveles de exposición a los plaguicidas anticolinesterásicos, que a su vez se reflejó en la amplia variación de los valores del indicador de exposición.

3. La sintomatología presente en los fumigadores estudiados estuvo relacionada con la exposición a los plaguicidas anticolinesterásicos.

\section{REFERENCIAS BIBLIOGRÁFICAS}

1. Environ. Prot. Agency (USA). Pesticide Industry Sales and Usage: 1998 and 1999 Market Estimates. Washington, DC: USEPA. 2002.

2. Sullivan JB Jr., Blose J. Organophosphate and carbamate insecticides. In: Sullivan JB. Krieger GR, eds. Hazardous materials toxicology: clinical principles of environmental health. Baltimore. Maryland. United State of America: Williams and Wilkins. 1992; p. 1015-1026.

3. Kushik J, Chandrabhan D. Sources of exposure to and public health implications of organophosphate pesticide. Pan Am J Public Health 2003; 14 (3).

4. Hsieh BH, Deng JF, Ger J, Tsai WJ. Acetylcholinesterase Inhibition and the Extrapyramidal Syndrome: A Review of the Neurotoxicity of Organophosphate. Neurotoxicology 2001; 22: 423-427.

5. DeBleecker J, Van Den Neucker K, Colardyn F. Intermediate syndrome in organophosphorous poisoning: A prospective study. Crit Care Med 1993; 21: 1706-1711.

6. De Bleecker J, Van Den Neucker K, Willems J. The intermediate syndrome in organophosphate poisoning: Presentation of a case and review of the literature. J Clin Toxicol 1992; 30: 321-329.

7. Johnson M. Organophosphates an delayed neuropathy. Toxicol Appl Pharmacol 1990; 102: 385-389.

8. Lotti M, Caroldi S, Capodicasa E, Moretto A. Promotion of organophosphate-induced delayed polyneuropathy by phenylmethanesulfonyl fluoride. Toxicol Appl Pharmacol 1991; 108: 223-241.

9. Jamal GA, Hansen S, Julu POO. Low level exposures to organophosphorus esters may cause neurotoxicity. Toxicology 2002; 181-182: 23-33.

10. Ray DE. Richards PG. The potential for toxic effects of chronic low-dose exposure to organophosphates. Toxicology Lett 2001; 120: 343-351.

11. Steenland K, Jenkins B, Ames RG. Chronic neurological sequelae to organophosphate poisoning. Am J Public Health 1994; 84: 731-736.

12. Amorin LCA. Biomarkers for evaluating exposure to chemical agents present in the environment. Rev Bras Epidemiol 2003; 6 (supl 1).
13. Maroni M, Jarvisalo J, La Ferla L. The WHO. UNDP epidemiological study on the health effectcs of exposure to organophosphorus pesticides. Toxi col Lett 1986; 33: 115-123.

14. Ellman GL, Courtney KD, Andres V, Featherstone R.M. A new and rapid colorimetric determination of cholinesterase activity. Biochem Pharmacol 1961; 7: 88-95.

15. Helmick JyJ, Smith J. Guías para el tratamiento y la disposición de pequeñas cantidades de desechos de plaguicidas. OPS/OMS. Metapec, México. 1993. p. 1,38 .

16. Nigg HN, Stamper JH, Easter E, DeJonge JO: Protection afforded greenhouse pesticide applicators by coveralls: a field test. Arch Environ Contam Toxicol 1993; 25(4): 529-533.

17. Delgado O, Almirall, Sánchez R. Evaluación de funciones psicomotoras en trabajadores expuestos habitualmente a plaguicidas. Rev Cub Hig Epid 1986; 24(1): 103-110.

18. Stokes L Stark A, Marshall E, Narang A. Neurotoxicity among pesticide applicators exposed to organophosphates. Occup Environ Med 1995; 52(10): 648-653.

19. Gordon M, Richter ED. Hazards associated with aerial spraying of organophosphate insecticides in Israel. Rev Environ Health 1991; 9(4): 229-38.

20. Peedicayil J, Ennest K, Thomas M, Kanagasabapathy AS, Stephen PM. The effect of organophosphorus compounds on serum pseudocholinesterase levels in a group of industrial workers. Hum Exp Toxicol 1991; 10: 275-278.

21. Palacios-Nava ME, Paz-Román P, Hernández-Robles S, Mendoza-Alvarado L. Sintomatología persistente en trabajadores industrialmente expuestos a plaguìicidas organofosforados. Salud Pública Mex 1999; 41: 55-61.

22. Dyer SM, Catan M, Pisaniello DL, Williams FM, Edwards JW. Peripheral cholinesterase inhibition by occupational chlorpyrifos exposure in Australian termicide aplicators. Toxicology 2001; 169: 177185.

23. Stefanidou M, Athanaselis $\mathrm{S}$, Velonakis $\mathrm{M}$, Pappas F, Koutselinis A. Occupational exposure to cholinesterase inhibiting pesticides: a Greek case. International Journal of Environmental Health Research 2003; 13: 23-29.

24. He F. Biological monitoring of occupational pesticides exposure. Int Arch Occup Environ Health 1993; 65 (1 suppl.): S69-S76.

25. Strong LL, Thompson B, Coronado GD, Griffith WC, Vigoren EM, Islas I. Health symptoms and exposure to organophosphate pesticides in farmworkers. Am J Ind Med 2004; 46(6); 599-606. 